\title{
A Dual-band Low Frequency Metamaterial-inspired Magnetic-based Antennas
}

\author{
Chia-Ching Lin and Richard W. Ziolkowski \\ Department of Electrical and Computer Engineering \\ University of Arizona, Tucson, AZ 85721, USA \\ E-mail: cclin@ece.arizona.edu, ziolkowski@ece.arizona.edu
}

\section{Introduction}

Many applications have been proposed that use metamaterials (MTMs), artificial materials having engineered electromagnetic properties, to obtain a desired, but unusual electromagnetic behavior. For instance, metamaterial-inspired efficient electrically small antennas have been proposed which have led to an efficacious, electrically-small antenna design methodology and have drawn much attention [1]. The main innovation of those designs was the introduction of an electrically small, resonant metamaterial-inspired parasitic element in the very near field of an electrically small radiator. The metamaterial-inspired parasitic structure provides the means for the antenna system to be resonant (reactive matching) and resistive

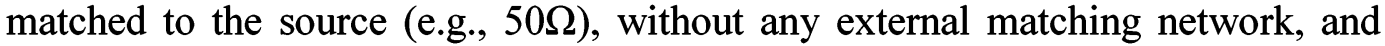
facilitates the efficient radiation of electromagnetic waves.

Recent technical advances have changed the emergency and military communications design specifications, especially the expectations for antennas. An efficient antenna system operating at multi-frequency bands would be an attractive design for many new applications which operate over the lower frequency bands. Synthesizing the unusual properties of MTMs and the ideas associated with lowering the operating frequencies of the magnetic-based EZ antennas [2], we have extended the three-dimensional (3D) magnetic-based EZ antenna designs to have multiple resonant frequencies in the VHF band. This paper introduces a dual-band, efficient, magnetic-based, 3D metamaterial-inspired antenna, i.e., a 3D dual-band magnetic-based EZ antenna. This EZ antenna system is naturally matched to the source at the desired frequency points; and thus, it is an efficient radiator at those frequencies. The performance of this 3D dual-band magnetic-based EZ antenna system was simulated with Ansoft's High Frequency Structure Simulator (HFSS). These HFSS-predicted performance characteristics for the design frequencies of interest are presented.

\section{Analysis and Design}

The 3D magnetic-based EZ antenna systems were first presented in [1]. They utilized the MTM-based antenna design concepts: an electrically small MTMinspired element, i.e., a 3D extruded capacitively loaded loop (CLL) which produces the needed MNG effects, is placed in the very near field of the driven magnetic dipole element, i.e., the electrically small semi-circular loop antenna 
which is coaxially fed through a finite perfect electric conductor (PEC) ground plane. The 3D CLL element produces the requisite capacitance to achieve the desired resonant behavior, i.e., because the reactance of the small loop antenna is inductive and the metamaterial-inspired element is capacitive, their combination can be designed to achieve a resonant $\mathrm{LC}$ system.

The design specifications of the dual-band 3D magnetic-based EZ antenna formed by two CLL elements are illustrated in Fig. 1. The proposed 3D magnetic-based antenna system produces the desired dual-band operation by combining two CLL elements: CLL element 1 and CLL element 2, with a semi-circular loop antenna coaxially-fed through a finite PEC ground plane. The CLL element 1, the first matched radiating element, is excited directly by the semi-loop antenna. It thus directly captures the magnetic flux generated by the small semi-circular loop antenna and then provides the necessary capacitance to achieve the first resonance point. The CLL element 2 , which is located in very close proximity to the CLL element 1 , is coupled to it by the strong magnetic flux produced by the driven element and the CLL element 1 . Being larger in size, it produces the desired resonant behavior at a much lower frequency. The manner in which the dual-band 3D magnetic-based EZ antenna operates is closely related to its single-band version, except for the mutual coupling between these two CLL elements.

The element length 1 , element height 1 , depth 1 , gap length 1 and stub height 1 that define CLL element 1 at the higher resonant frequency are $80 \mathrm{~mm}, 40 \mathrm{~mm}$, $12.5 \mathrm{~mm}, 0.75 \mathrm{~mm}$, and $3 \mathrm{~mm}$, respectively. Similarly, the corresponding dimensions of CLL element 2, which provides the lower resonant frequency, are the element length 2 , element height 2 , depth 2 , gap length 2 and stub height 2 , which are equal to $80 \mathrm{~mm}, 40 \mathrm{~mm}, 52 \mathrm{~mm}, 0.75 \mathrm{~mm}$ and $3 \mathrm{~mm}$, respectively. Additionally, the gap in each CLL element is filled with a dielectric material, quartz. Because this increases the capacitances, the system can be made to be resonant at even lower frequencies. The reason for the selection of quartz as the dielectric material is that it has a high dielectric constant value (3.78) and a low dielectric loss tangent $\left(1 \times 10^{-4}\right)$. Additionally, since it is not easy to achieve the desired orientations and dimensions of the CLL element once it is laser welded to the ground plane, the presence of the quartz slab ensures that the two arms of the CLL element have the desired positions and relative orientations. Thus, not only does the quartz spacer provide the desired capacitance, it also enhances the mechanically stability of the fabricated structure. The CLL separation, antenna radius, and wire radius are $4.15 \mathrm{~mm}, 14.5 \mathrm{~mm}$ and $2 \mathrm{~mm}$, respectively. The center of the semi-circular loop antenna is displaced $-3 \mathrm{~mm}$ along y-axis compared to the location of the CLL element 1 to obtain a better input impedance match at the design frequencies. Figure 2 gives the HFSS-predicted dual-band resonant behavior. The return loss is $-23.2314 \mathrm{~dB}$ and $-25.5404 \mathrm{~dB}$ at $282.8 \mathrm{MHz}$ and $491.35 \mathrm{MHz}$, respectively. Additionally, the corresponding radiation efficiencies for these two resonance frequencies are $97.9 \%$ and $70.4 \%$, respectively. The higher resonant frequency is dominated by the properties of CLL element 1 since its size is much smaller than that of CLL element 2 . This is also the reason why 
the radiation efficiency is lower at the higher resonance frequency as compared to its value at the lower resonance frequency. The asymmetric pair of CLL elements plays a critical role in the performance of the overall antenna system. They not only expedite the internal impedance matching, but they also act as part of the radiator, which in turn facilitates an increase in the overall radiation efficiency.

A highly tunable dual-band frequency antenna is desirable because there are a variety of low frequency applications. Therefore, it is desirable to define an efficient and systematic way to tweak the design of the structure in order to achieve the desired resonant frequencies. The parameters which are the major factors that produce a fine tuning of the performance of the overall antenna system are the separation between the CLL elements and the stub height and depth. For instance, one can see from Fig. 2 that the low and high resonance frequencies come, respectively, from CLL element 2 and CLL element 1. If one wants to make the two resonance frequency points get closer to each other, one can decrease the depth 2 and increase the CLL separation in order to reduce the capacitance between the CLL elements. This will increase the corresponding resonant frequency. Another variation is to increase the depth 1 to get a larger size of the CLL element 1 so that its capacitance values will increase and, hence, further decrease the resonance frequency. Notice that as one alters the size of the CLL elements, the two resonance frequencies will change simultaneously because they depend on the mutual coupling between these two CLL elements. The stub heights provide the major capacitances of this antenna; it can be further matched to the reactance part of the semi-circular loop antenna. Consequently, one can design the CLL elements and the driven element to achieve resonant frequencies which are close to any desired frequency points. Figure 3 shows a case for which the system was tuned to produce much closer resonant frequencies.

\section{Conclusion}

Dual-band magnetic-based 3D antennas were introduced and shown to achieve efficient radiating elements that operate over a low frequency band. The resonant frequencies of the antenna system can be designed for a broad range of frequencies by tuning separately the near field resonant parasitic elements. We are continuing our investigations to determine if other structural variations can further reduce the return losses at the lower VHF frequencies or could better control the separation between the two resonance frequencies.

\section{References}

[1] A. Erentok and R. W. Ziolkowski, "Metamaterial-inspired efficient electrically-small antennas," IEEE Trans. Antennas Propag., vol. 56, no..3, pp. 691-707, March 2008

[2] R. W. Ziolkowski and Chia-Ching Lin, "Metamaterial-Inspired MagneticBased UHF and VHF Antennas," IEEE Antennas Propag. Symposium, July 2008. 

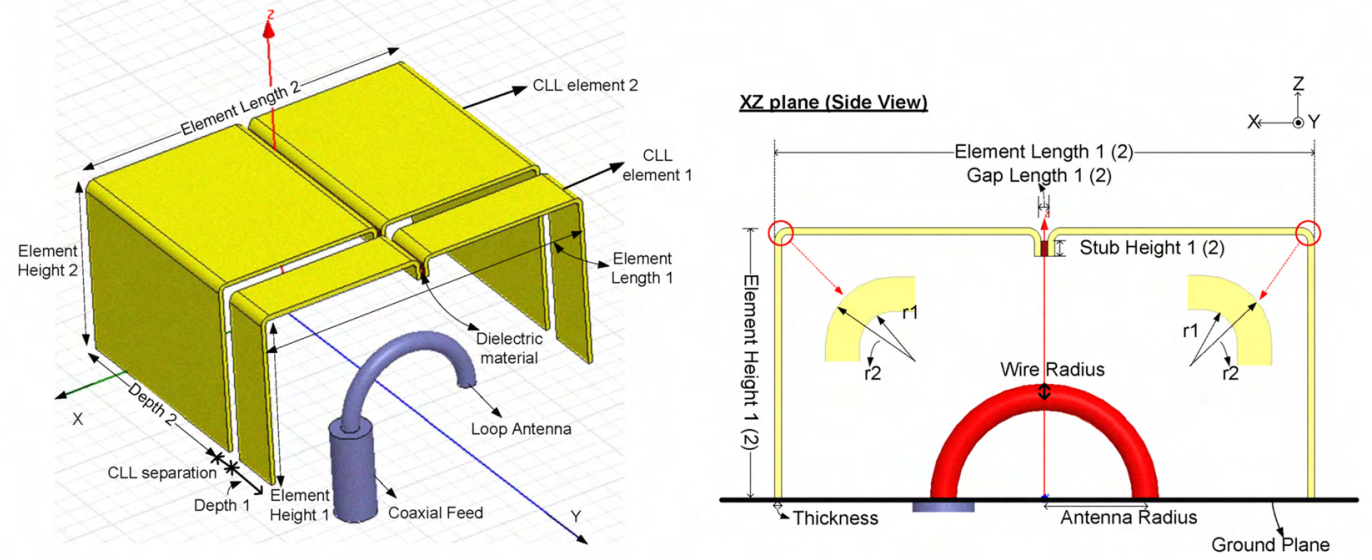

Fig.1: The geometry of the dual-band 3D magnetic-based metamaterial-inspired antenna and the detailed specifications of each design variable

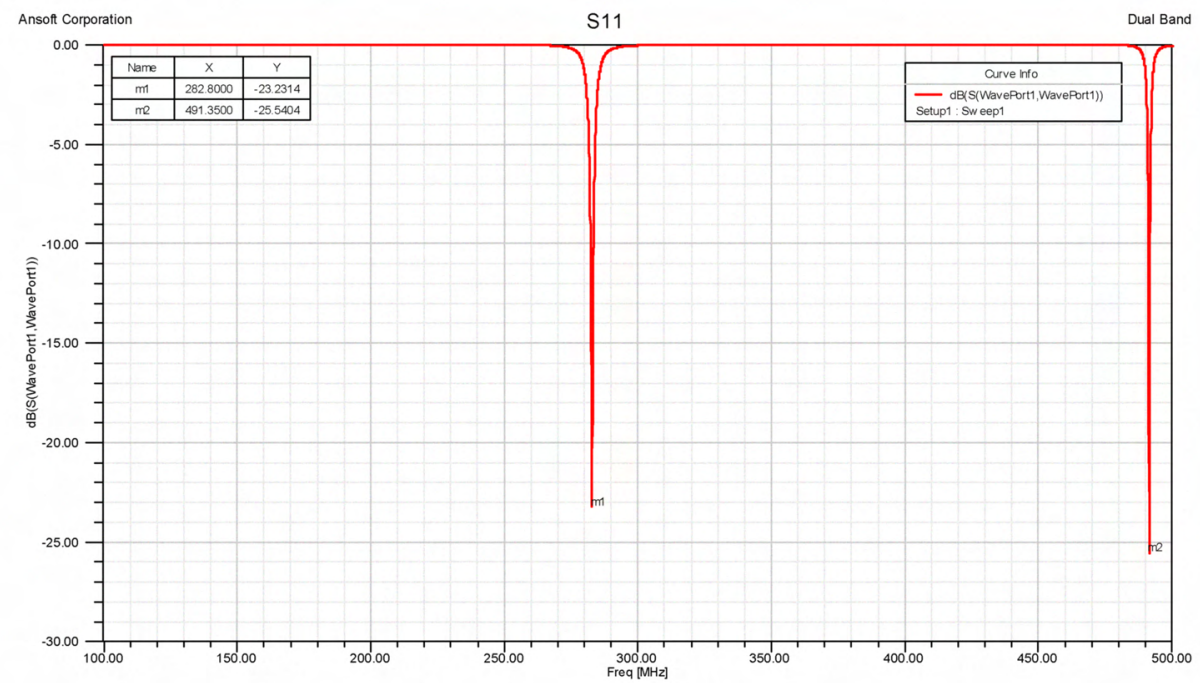

Fig. 2: HFSS predicted $\mathrm{S}_{11}$ values for the dual-band 3D magnetic-based metamaterial-inspired antenna with widely spaced resonance frequencies

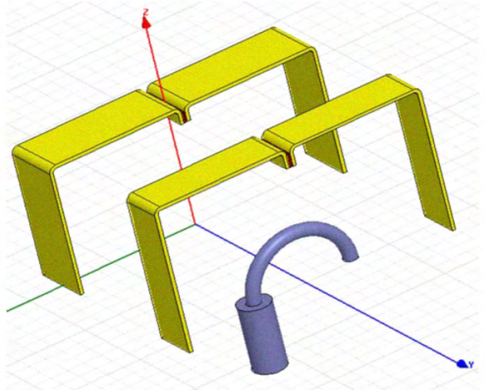

(a)

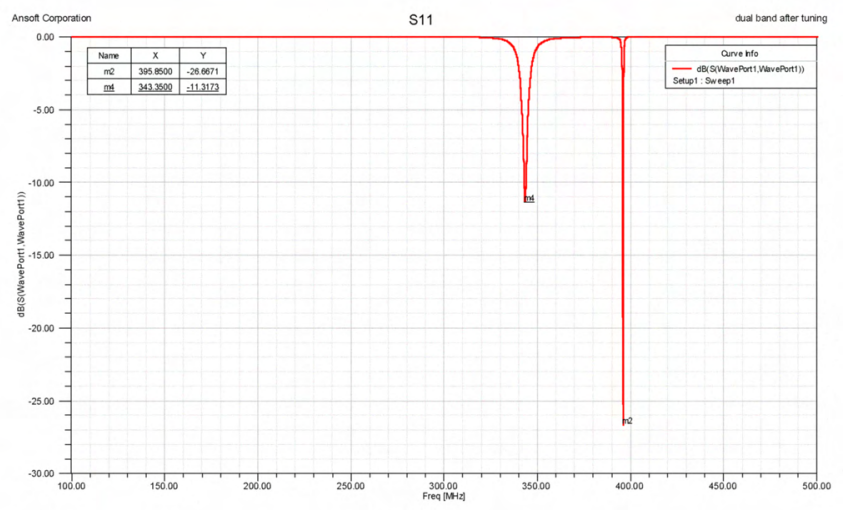

(b)

Fig. 3: (a) Dual-band 3D magnetic-based metamaterial-inspired antenna with closely spaced resonance frequencies, (b) HFSS predicted $S_{11}$ values 